CONFORMAL GEOMETRY AND DYNAMICS

An Electronic Journal of the American Mathematical Society

Volume 11, Pages 12-28 (March 1, 2007)

S $1088-4173(07) 00158-0$

\title{
ON REFLECTIONS IN JORDAN CURVES
}

\author{
OLE JACOB BROCH
}

\begin{abstract}
A purely geometric method for constructing reflections in Jordan curves on the Riemann sphere based on hyperbolic geodesics is introduced. It is then possible to investigate the relations between the geometry of a Jordan domain $D$ and the properties of the reflection by studying properties of hyperbolic geodesics. This idea is used to characterize unbounded Jordan John domains in terms of reflections satisfying a kind of Lipschitz condition.
\end{abstract}

\section{INTRODUCTION}

A domain $D \subset \overline{\mathbb{R}^{2}}$ admits a reflection in its boundary $\partial D$ if there exists a homeomorphism $f$ of $\bar{D}$ such that

$$
\begin{aligned}
& f(D)=D^{*} \text { where } D^{*}=\overline{\mathbb{R}^{2}} \backslash \bar{D}, \text { and } \\
& f(z)=z \text { for } z \in \partial D .
\end{aligned}
$$

This is the definition given by Gehring and Hag [11. For instance, the map defined by

$$
g\left(r e^{i \vartheta}\right)=r^{-2} e^{i \vartheta}, r \neq 0 ; \quad g(0)=\infty, \quad g(\infty)=0
$$

is a reflection in $\mathbb{T}$, the boundary of the unit disk $\mathbb{D}=\{z:|z|<1\}$. This is an artificial example, but it emphasizes that the definition used here does not require $f(f(z))=z$. Note that if $f$ is only defined in $\bar{D}$, then one may define a reflection $F: \overline{\mathbb{R}^{2}} \rightarrow \overline{\mathbb{R}^{2}}$ by $\left.F\right|_{\bar{D}}=f$ and $\left.F\right|_{D^{*}}=f^{-1}$, and then one has $F(F(z))=z$.

A reflection $f$ is said to have the property $P$ if its restriction to $D$ has that property. For example: $f$ is quasiconformal if $\left.f\right|_{D}$ is quasiconformal. Ahlfors considered quasiconformal reflections in [1, along with hyperbolic and Euclidean biLipschitz reflections (see also [2], 11] and [20]). Another kind of reflection was studied by Earle and Nag [7], who used the Douady-Earle extension [6] to construct reflections rather than the Beurling-Ahlfors extension [3, which was Ahlfors' means of constructing them. Later still, Gehring and Hag proved the appealing result, that a domain $D$ admits a reflection in its boundary if and only if it is a Jordan domain (see [11, Theorem 1.2]). Thus little is lost by considering only Jordan domains in the following.

In this paper we exhibit and study a particular choice of reflection $R_{D}$ for each Jordan domain $D$. This reflection is constructed using hyperbolic lines, and its properties (like conformality and quasiconformality) literally reflect the geometry of the domain. The construction is reminiscent of the "usual" way of constructing reflections in lines. The construction is given in Section 4. In Section 5] we will

Received by the editors August 24, 2006.

2000 Mathematics Subject Classification. Primary 30C20; Secondary 30C99.

Key words and phrases. John domain, reflection, hyperbolic geodesic.

(C)2007 American Mathematical Society Reverts to public domain 28 years from publication 
see some new examples of how the properties of the reflection correspond to the geometry of the domain. The main result of this paper is Theorem 5.5, which characterizes John disks in terms of reflections satisfying a kind of Lipschitz condition. This result is interesting because it is rarely possible to compare the inside of a John disk with the outside, without getting a quasidisk.

We also give a new proof for a result about quasidisks due to Ahlfors [2] (see Theorem 5.10 below).

In Section 6 we complement some of the material in Sections 4 and 5 by giving examples and remarks, in addition to a restatement of the main result.

Section 3 contains auxiliary material on the geometry of hyperbolic geodesics. These results are "one sided" versions of results for uniform domains and quasidisks, and may be interesting in their own right. In particular, we obtain new characterizations of John disks in terms of certain minimum properties of hyperbolic geodesics.

Section 2 contains basic definitions, notation and results that will be used.

\section{Preliminaries}

Here we collect some basic definitions and results that will be needed throughout.

In this paper, unless stated otherwise, $D$ will denote a Jordan domain in $\overline{\mathbb{R}^{2}}$, and $D^{*}=\overline{\mathbb{R}^{2}} \backslash \bar{D}$ will be its complementary domain. A domain $D$ is a Jordan domain if $\partial D \subset \overline{\mathbb{R}^{2}}$ is a Jordan curve. Note that $\partial D$ will be used for the common boundary of $D$ and $D^{*}$. Hyperbolic segments or lines in $D$, possibly with one or both of the end points in $\partial D$, will always be denoted by $\gamma$. To be more specific, $\gamma\left[z_{1}, z_{2}\right]$ will be a hyperbolic segment or line from $z_{1}$ to $z_{2}$ in $\bar{D}$. Likewise $\gamma^{*}$ denotes a hyperbolic segment in $D^{*}$. Thus if $\zeta_{1}, \zeta_{2} \in \partial D=\partial D^{*}, \gamma\left[\zeta_{1}, \zeta_{2}\right]$ is a hyperbolic line in $D$ and $\gamma^{*}\left[\zeta_{1}, \zeta_{2}\right]$ is a hyperbolic line in $D^{*}$. If $\alpha$ is any arc in $D\left(\right.$ or $\left.D^{*}\right)$ and $z_{1}, z_{2} \in \alpha$, then $\alpha\left[z_{1}, z_{2}\right]$ will denote the subarc of $\alpha$ from $z_{1}$ to $z_{2}$.

The collective term geodesic or hyperbolic geodesic will sometimes be used for hyperbolic lines, segments and rays alike. It will be clear, from the context, in which of these classes the arc belongs. Geodesic line will always mean a geodesic with both end points in the boundary of the domain in question.

Possible uses of the reflection procedure will be demonstrated by studying John disks and quasidisks. Recall that a $K$-quasidisk is the image of a disk or a half plane under a $K$-quasiconformal self homeomorphism of $\overline{\mathbb{R}^{2}}$. A simply connected domain $D \subset \overline{\mathbb{R}^{2}}$ with at least two boundary points is a $c$-John disk if any two points $z_{1}, z_{2}$ in $D \backslash\{\infty\}$ may be joined by a rectifiable arc $\alpha$ in $D \backslash\{\infty\}$ with

$$
\min _{j=1,2} \ell\left(\alpha\left[z_{j}, z\right]\right) \leq c \operatorname{dist}(z, \partial D)
$$

for every $z \in \alpha$; here $\ell(\alpha)$ denotes the length of $\alpha$ and $\operatorname{dist}(z, A)=\inf _{w \in A}|z-w|$. All quasidisks are John disks 21.

In general John disks do not have to be Jordan domains, since $\mathbb{D} \backslash[0,1)$ is a John disk. This is why we will constantly make the additional assumption that $D$ be a Jordan domain. We will sometimes talk about the center of a John disk. By [21, Theorem 2.14] the definition of a John disk used in the present paper implies the existence of a point $x_{0} \in \bar{D}$, called a center of $D$, such that any point $x_{1} \in D$ may be joined to $x_{0}$ by a rectifiable arc $\alpha$ such that for every $x \in \alpha$ we have $\ell\left(\alpha\left[x, x_{1}\right]\right) \leq c \operatorname{dist}(x, \partial D)$. Here $c$ may be different from the constant in (2.1). If $D$ is unbounded, then $x_{0}=\infty$ [21, Remark 2.3.2]. 
A c-linearly connected domain is a domain $D$ where any pair $z_{1}, z_{2}$ of points may be joined by an $\operatorname{arc} \alpha$ with

$$
\operatorname{diam} \alpha \leq c\left|z_{1}-z_{2}\right| .
$$

In both John disks and linearly connected domains we may replace the $\operatorname{arcs} \alpha$ by hyperbolic segments (see [13, Theorem 4.1], 21, Theorem 5.2] and [22, Proposition 5.6]). A Jordan domain $D$ is a John disk if and only if $D^{*}$ is linearly connected [21, Theorem 4.5].

For a Jordan domain $D$, we will let $\partial_{r} D$ denote the set of rectifiably accessible boundary points, while we write $D_{r}=D \cup \partial_{r} D$. If $D$ is a John domain, then $\partial_{r} D=\partial D$ [21, Remark 6.6]. In any case, $\partial_{r} D$ is always dense in $\partial D$. By $\lambda_{D}\left(z_{1}, z_{2}\right)$ we mean the internal length distance between $z_{1}, z_{2} \in D_{r}$ :

$$
\lambda_{D}\left(z_{1}, z_{2}\right)=\inf \left\{\ell(\beta): \beta \text { is a rectifiable arc joining } z_{1} \text { and } z_{2} \text { in } D\right\}
$$

(see e.g. Section 3 in [21] or Section 2 in [5]).

A convention: Throughout this paper we will use $\tilde{z}$ for the preimage in $\overline{\mathbb{H}}(\overline{\mathbb{D}})$ of any $z \in D$ under a conformal map $\varphi: \mathbb{H} \rightarrow D(\varphi: \mathbb{D} \rightarrow D)$ or its extension to the closures $\varphi: \overline{\mathbb{H}} \rightarrow \bar{D}(\varphi: \overline{\mathbb{D}} \rightarrow \bar{D})$. Here $\mathbb{H}$ is the upper half plane. Whenever we have a conformal map, we always extend it as a homeomorphism in this way, and use the same letter for the extended function. This extension is possible due to the Osgood-Carathéodory extension theorem (see e.g. [22, Theorem 2.6]).

In order to simplify notation, we will sometimes write $f g=f(g)$ for the composition of two maps $f$ and $g$ (whenever it is defined).

We will need some results which are quoted here for later reference.

Theorem 2.3 ([21], Theorem 5.6). Let $D \subset \overline{\mathbb{R}^{2}}$ be a c-John disk with center $z_{0}$, with $z_{0}=\infty$ if $D$ is unbounded. Let $\gamma$ be a hyperbolic geodesic from $z_{0}$ to $\partial D$. Then

$$
\ell\left(\gamma\left[z_{1}, z_{2}\right]\right) \leq c_{1}\left|z_{1}-z_{2}\right|
$$

for all $z_{1}, z_{2} \in \gamma$, where $c_{1}=c_{1}\left(c, z_{0}\right)$.

Remember that a homeomorphism $\varphi:(X, \varrho) \rightarrow(Y, \sigma)$ between two metric spaces is $\eta$-quasisymmetric if there is a homeomorphism $\eta:[0, \infty) \rightarrow[0, \infty)$ such that

$$
\frac{\sigma(f(a), f(b))}{\sigma(f(b), f(c))} \leq \eta\left(\frac{\varrho(a, b)}{\varrho(b, c)}\right)
$$

for all $a, b, c \in X, b \neq c$. (See the paper by Tukia and Väisälä [24] or Heinonen's book [17] for the basic theory about quasisymmetric maps.)

Theorem 2.4 ([21], [5]). A Jordan domain $D \subset \overline{\mathbb{R}^{2}}$ with $\infty \in \partial D$ is a c-John disk if and only if every conformal map $\varphi: \mathbb{H} \rightarrow D$ with $\varphi(\infty)=\infty$ extends as a homeomorphism $\varphi: \overline{\mathbb{H}} \rightarrow \bar{D}$ such that $\varphi: \overline{\mathbb{H}} \rightarrow\left(D_{r}, \lambda_{D}\right)$ is $\eta$-quasisymmetric, where $\eta$ and $c$ depend only on each other.

The Gehring-Hayman Theorem 2.5 (14). Let $D$ be a simply connected domain in $\mathbb{R}^{2}$, and let $z_{1}, z_{2} \in D_{r}$. If $\gamma$ is the hyperbolic segment from $z_{1}$ to $z_{2}$ in $D$ and $\alpha$ is any Jordan arc from $z_{1}$ to $z_{2}$ in $D$, we have that

$$
\ell(\gamma) \leq K \ell(\alpha),
$$

where $K$ is a universal constant.

A proof may also be found in [22], pp. 88-90. 


\section{Geometric properties of hyperbolic GeOdesics}

In this section we will prove results about hyperbolic geodesics that will be used in Section 5, Remember that $\gamma\left[z_{1}, z_{2}\right]$ denotes the hyperbolic segment, ray or line from $z_{1}$ to $z_{2}$ whenever $z_{1}$ and $z_{2}$ lie in (the closure of) a simply connected plane domain.

The point of departure is the following result of Gehring and Hag, which says that a simply connected proper subdomain $D$ of $\mathbb{R}^{2}$ is a quasidisk if and only if hyperbolic geodesics in $D$ have the min-max property.

Theorem 3.1 ([10], Corollary 2.10). A simply connected proper subdomain $D \subset \mathbb{R}^{2}$ is a K-quasidisk if and only if there is a constant $c$ such that for all $z_{1}, z_{2} \in D$,

$$
\frac{1}{c} \min _{j=1,2}\left|z_{j}-w\right| \leq|z-w| \leq c \max _{j=1,2}\left|z_{j}-w\right|
$$

for any $z \in \gamma\left[z_{1}, z_{2}\right]$ and every $w \notin D$. The constants $K$ and $c$ depend only on each other.

Remark 3.3. If $D$ is a quasidisk, then all boundary points are rectifiably accessible, and a limiting procedure shows that (3.2) also holds if one or both of $z_{1}$ and $z_{2}$ lie in $\partial D$. The rectifiable accessibility of the boundary points of a quasidisk follows from e.g. 21, Theorem 9.2 and Remark 6.6.

We will explain how the two inequalities of Theorem 3.1 break into two separate "one sided" characterizations of John and linearly connected domains. Most of the arguments in the present section are, in one way or another, adapted from or inspired by arguments in [10], which the author hereby acknowledges. We have chosen to include proofs, for completeness and clarity.

The following characterization of John disks in terms of a minimum property of hyperbolic geodesics represents a one sided analogue to Theorem 3.1 and might be interesting in its own right.

Theorem 3.4. Suppose that $D$ is a Jordan domain in $\overline{\mathbb{R}^{2}}$. Then $D$ is an a-John disk if and only if there is a constant $c$ such that for $z_{1}, z_{2} \in D_{r}$ and every metric $d$ with $|z-w| \leq d(z, w) \leq \lambda_{D}(z, w)$ we have

$$
\min _{j=1,2} d\left(z_{j}, w\right) \leq c d(z, w)
$$

for every $w \in \partial_{r} D$ and every $z \in \gamma\left[z_{1}, z_{2}\right]$.

Proof. First we note that if $d$ is a metric with $|z-w| \leq d(z, w) \leq \lambda_{D}(z, w)$, then the length with respect to the $d$ metric of any rectifiable arc is the same as the Euclidean length.

Let $D$ be an $a$-John disk. Take $z_{1}, z_{2} \in \bar{D}$ and let $z \in \gamma\left[z_{1}, z_{2}\right], w \in \partial D$. Then we have

$$
\begin{aligned}
\min _{j=1,2} d\left(z_{j}, w\right) & \leq \min _{j=1,2} d\left(z_{j}, z\right)+d(z, w) \\
& \leq \min _{j=1,2} \ell\left(\gamma\left[z_{j}, z\right]\right)+d(z, w) \\
& \leq a \operatorname{dist}(z, \partial D)+d(z, w) \\
& \leq a|z-w|+d(z, w) \\
& \leq(a+1) d(z, w)
\end{aligned}
$$


where the third inequality follows from the definition of an $a$-John disk. We also used the fact that hyperbolic geodesics $\gamma$ can be used in (2.1).

Now suppose that (3.5) holds in $D$. Then, in particular, (3.5) holds for $\lambda_{D}$. Let $z_{1}, z_{2} \in D$ and take $z \in \gamma\left[z_{1}, z_{2}\right]$. Let $w \in \partial D$ such that $|z-w|=\operatorname{dist}(z, \partial D)$. Then $w \in \partial_{r} D$ and $\lambda_{D}(z, w)=|z-w|=\operatorname{dist}(z, \partial D)$. By using the Gehring-Hayman Theorem 2.5 together with (3.5), we have:

$$
\begin{aligned}
\min _{j=1,2} \ell\left(\gamma\left[z_{j}, z\right]\right) & \leq \min _{j=1,2} K \lambda_{D}\left(z_{j}, z\right) \\
& \leq K\left(\min _{j=1,2} \lambda_{D}\left(z_{j}, w\right)+\lambda_{D}(z, w)\right) \\
& \leq K\left(c \lambda_{D}(z, w)+\lambda_{D}(z, w)\right) \\
& =K(c+1) \lambda_{D}(z, w)=K(c+1) \operatorname{dist}(z, \partial D) .
\end{aligned}
$$

Thus $D$ is an $a=K(c+1)$-John disk by definition (2.1).

Remark 3.6. It is not necessary to assume $D$ to be a Jordan domain in order to prove the equivalence in the above theorem. The only assumption needed is that $D$ be simply connected and of hyperbolic type. For the proof, it was convenient to assume that $D$ was a Jordan domain in order to avoid certain technicalities regarding $\partial_{r} D$.

A simply connected Jordan domain is a quasidisk if and only if it is at the same time a John disk and a linearly connected domain [21, Theorem 9.3], [22, Theorem 5.9]. Therefore it should not be surprising that we may characterize linearly connected domains in terms of a maximum property for hyperbolic geodesics.

Theorem 3.7. A simply connected domain $D$ in $\mathbb{R}^{2}$ is a-linearly connected if and only if there is a constant $b$ such that for any $z_{1}, z_{2} \in \bar{D}$ and any $w \notin D$,

$$
|z-w| \leq b \max _{j=1,2}\left|z_{j}-w\right|
$$

for every $z \in \gamma\left[z_{1}, z_{2}\right]$. The constants a and $b$ depend only on each other.

Proof. Once again the proof is adapted from [10, Theorem 2.7].

Let $D$ be $a$-linearly connected. According to [22, Proposition 5.6] condition (2.2) holds for hyperbolic geodesics in $D$ from $z_{1}$ to $z_{2}$ in $\bar{D}$. Let $z_{1}, z_{2} \in \bar{D}$. Take $z \in \gamma=\gamma\left[z_{1}, z_{2}\right]$ and any $w \in \mathbb{R}^{2} \backslash D$. Then

$$
\begin{aligned}
|z-w| & \leq \frac{1}{2}\left(\left|z_{1}-w\right|+\left|z_{1}-z\right|+\left|z_{2}-w\right|+\left|z_{2}-z\right|\right) \\
& \leq \frac{1}{2}\left(2 \operatorname{diam} \gamma+\left|z_{1}-w\right|+\left|z_{2}-w\right|\right) \\
& \leq \frac{1}{2}\left(2 a\left|z_{1}-z_{2}\right|+\left|z_{1}-w\right|+\left|z_{2}-w\right|\right) \\
& \leq \frac{1}{2}(2 a+1)\left(\left|z_{1}-w\right|+\left|z_{2}-w\right|\right) \\
& \leq(2 a+1) \max _{j=1,2}\left|z_{j}-w\right| .
\end{aligned}
$$

Conversely, suppose that $D$ has the maximum property for hyperbolic geodesics. Take $z_{1}, z_{2} \in D$. If $\left|z_{1}-z_{2}\right|<\operatorname{dist}\left(z_{1}, \partial D\right)$, then $z_{1}$ and $z_{2}$ may be joined by 
$\left[z_{1}, z_{2}\right] \subset D$. Otherwise, $\left|z_{1}-z_{2}\right| \geq \operatorname{dist}\left(z_{1}, \partial D\right)$. Then take $w \in \partial D$ with $\left|z_{1}-w\right|=$ $\operatorname{dist}\left(z_{1}, \partial D\right)$. Let $\gamma$ be the hyperbolic geodesic joining $z_{1}$ and $z_{2}$. If $z \in \gamma$, then

$$
|z-w| \leq b \max _{j=1,2}\left|z_{j}-w\right| \leq b\left(\left|z_{1}-w\right|+\left|z_{1}-z_{2}\right|\right) \leq 2 b\left|z_{1}-z_{2}\right|,
$$

which means that diam $\gamma \leq 4 b\left|z_{1}-z_{2}\right|$.

\section{Constructing Reflections}

We will mostly consider Jordan domains whose boundaries contain the point at infinity, but start by constructing reflections in the case when $\partial D \subset \mathbb{R}^{2}$. What we will do is to define a coordinate system in $D$ by means of hyperbolic geodesics.

Throughout this section $D$ will be a Jordan domain in $\overline{\mathbb{R}^{2}}$.

4.1. Reflection when $\partial D$ is bounded. Fix any point $p$ in $\partial D$. For $z$ in $D$ consider the full geodesic line in $D$ from $p$ through $z$. This line meets $\partial D$ again in some point $\xi \neq p$. Next consider the unique geodesic through $z$ that meets $\gamma[p, \xi]$ orthogonally; see Figure 1. This geodesic line will have two end points $\zeta^{1}$ and $\zeta^{2}$ in $\partial D$; number them according to orientation. Thus

$$
\{z\}=\gamma[p, \xi] \cap \gamma\left[\zeta^{1}, \zeta^{2}\right] .
$$

The triple $\left(\zeta^{1}, \xi, \zeta^{2}\right)_{p}$ will be called geodesic coordinates for $z$ with respect to $p$. Note that not any triple of points in $\partial D$ will do as geodesic coordinates for some point, but whenever $z$ is represented by such a triple we will write

$$
z=\left(\zeta^{1}, \xi, \zeta^{2}\right)_{p}
$$

Now the geodesics from $\zeta^{1}$ to $\zeta^{2}$ and from $p$ to $\xi$ in $D^{*}=\overline{\mathbb{R}^{2}} \backslash \bar{D}$ meet in a unique point $w \in D^{*}$; see the proof of Theorem 4.2 below. We define the reflection of $z$ as:

$$
R_{D, p}(z)=\left\{\begin{array}{l}
\gamma^{*}[p, \xi] \cap \gamma^{*}\left[\zeta^{1}, \zeta^{2}\right], \text { for } z \in D \\
z, \text { for } z \in \partial D
\end{array}\right.
$$

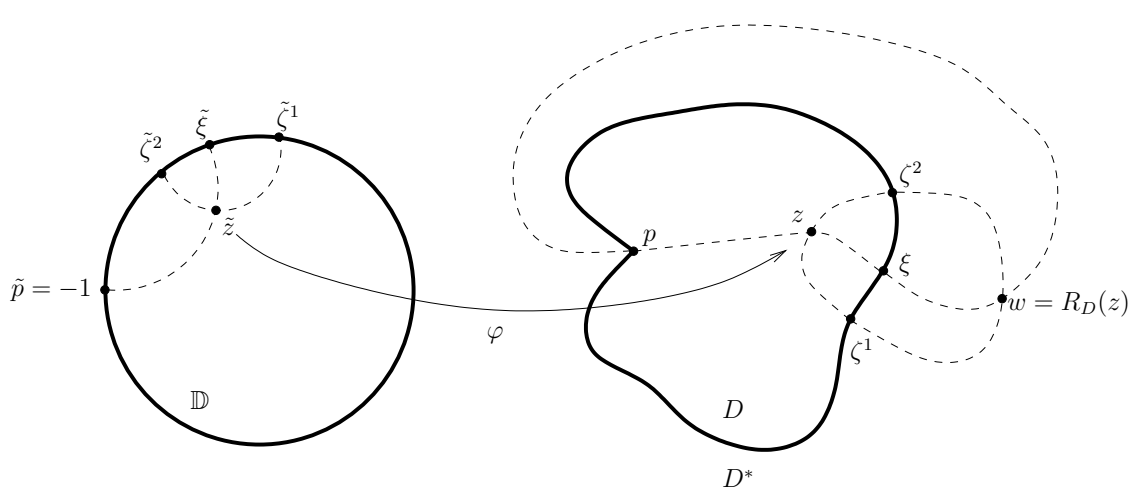

Figure 1. Constructing $R_{D, p}$ when $\partial D$ is bounded.

Note that there is some point $z_{0}$ in $D$ with $R_{D, p}\left(z_{0}\right)=\infty$. Also be aware that even if $w=R_{D, p}(z)$ with $z=\left(\zeta^{1}, \xi, \zeta^{2}\right)_{p}$, it is not true in general that $\left(\zeta^{1}, \xi, \zeta^{2}\right)_{p}$ are geodesic coordinates for $w$ in $D^{*}$. 
4.2. Reflection when $\infty \in \partial D$. When $\infty \in \partial D$, it is convenient to take $p=\infty$, and we leave $p=\infty$ out of the notation in this case. The construction is exactly the same as in the bounded case, except that it is also convenient to make reference to a conformal map $\varphi: \mathbb{H} \rightarrow D$ fixing $\infty$. If $\left(\zeta^{1}, \xi, \zeta^{2}\right)$ are geodesic coordinates for $z \in D$, then the geodesics in $\mathbb{H}$ joining $\tilde{\zeta}^{1}=\varphi^{-1}\left(\zeta^{1}\right)$ and $\tilde{\zeta}^{2}=\varphi^{-1}\left(\zeta^{2}\right)$ will be semicircles, while $\varphi^{-1}([\infty, \xi])$ is the bisector of each such half circle. In particular, $\left|\tilde{\zeta}^{1}-\tilde{\xi}\right|=\left|\tilde{\zeta}^{2}-\tilde{\xi}\right|$ (see Figure 2). Define the reflection of $z$ in $\partial D$ as follows.

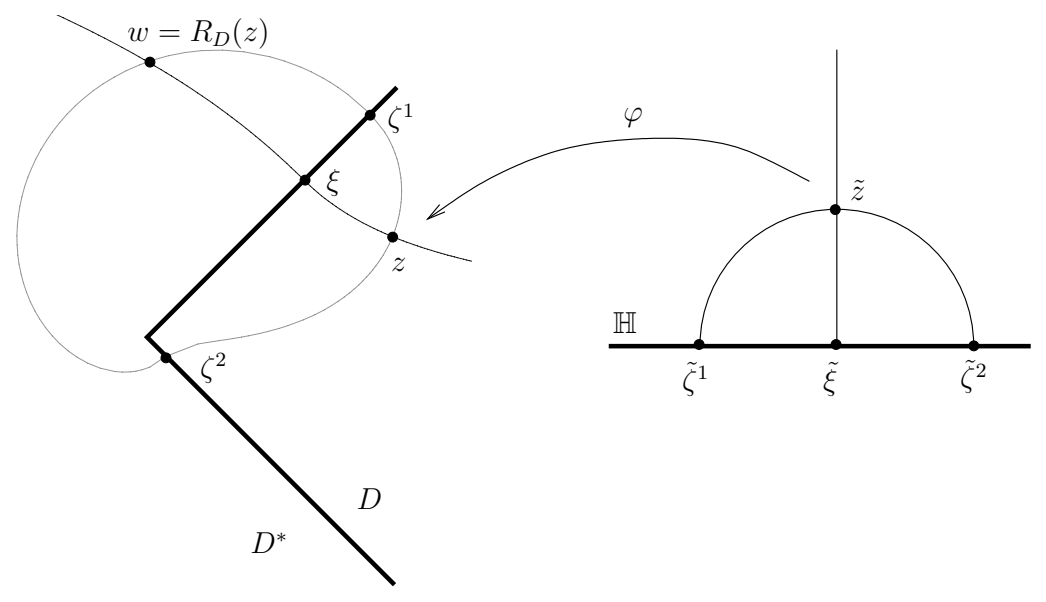

Figure 2. Constructing $R_{D}$ when $\infty \in \partial D$.

$$
w=R_{D}(z)=\left\{\begin{array}{l}
\gamma^{*}[\infty, \xi] \cap \gamma^{*}\left[\zeta^{1}, \zeta^{2}\right], \text { for } z \in D \\
z, \text { for } z \in \partial D \\
\infty, \text { for } z=\infty
\end{array}\right.
$$

Note that $R_{D, p}$ and $R_{D}$ are independent of any conformal maps involved.

Example 4.1. If $D$ is a half plane, $R_{D}$ corresponds to the usual reflection in a line. Thus when $D=\mathbb{H}, R_{D}(z)=\bar{z}$ (complex conjugation).

In Section 5 we will present examples of reflections in boundaries of quasidisks and John disks. In the meantime, see Figures 2 and 3 for actual reflections $R_{D}$ in the boundary of a sector and the half strip, respectively. The sector domain $D=\left\{z \in \mathbb{R}^{2}:|\arg z|<\pi / 4\right\}$ in Figure 2 is a quasidisk ([1]), while the outside of the half strip in Figure 3 is a John disk.

Next we confirm that $R_{D}$ and $R_{D, p}$ are indeed reflections, i.e. that they are homeomorphisms and fix each boundary point.

Theorem 4.2. Let $D$ be a Jordan domain with $\infty \in \partial D$. Then $R_{D}$ is a reflection.

Proof. We simply check the properties of $R_{D}$. This is, unfortunately, a bit tedious. Recall the notation from the construction of $R_{D}$. Let $\varphi: \mathbb{H} \rightarrow D$ and $\psi: \mathbb{H} \rightarrow D^{*}$ be conformal maps with $\varphi(\infty)=\psi(\infty)=\infty$.

First we show that $R_{D}$ is well defined. Take $z \in D$ with $z=\left(\zeta^{1}, \xi, \zeta^{2}\right)$. Then $C=\gamma[\infty, \xi] \cup \gamma^{*}[\infty, \xi]$ is a Jordan curve, and $\zeta^{1}, \zeta^{2}$ lie in different components of $\mathbb{R}^{2} \backslash C$. Then $\gamma^{*}\left[\zeta^{1}, \zeta^{2}\right]$ must intersect $C$ in $D^{*}$, since $\gamma^{*}\left[\zeta^{1}, \zeta^{2}\right] \subset D^{*}$. The 


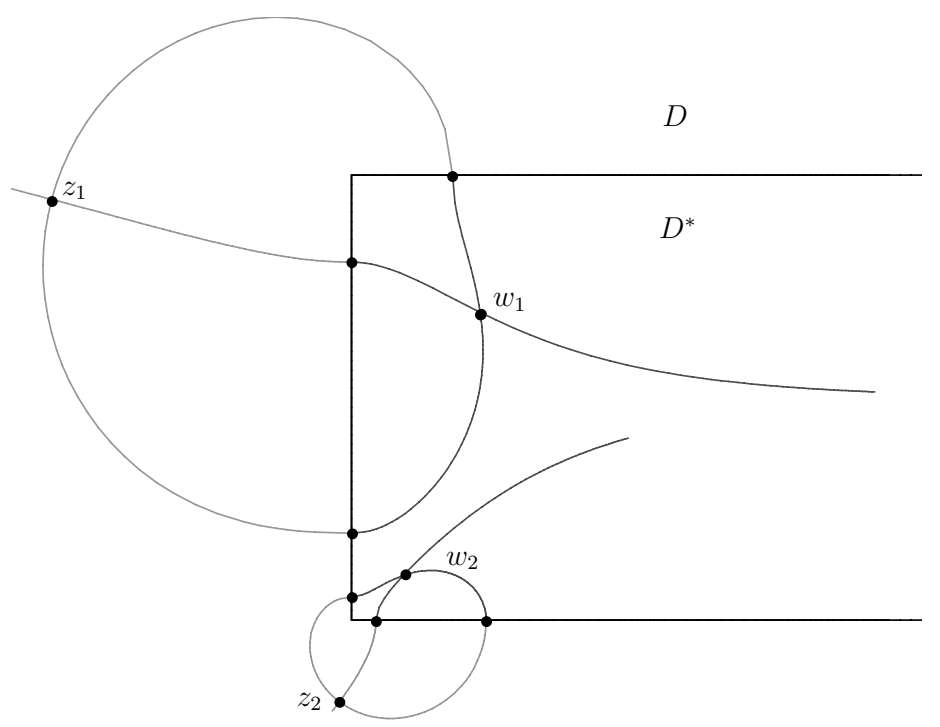

Figure 3. An actual example of $R_{D}$ when $D=\{z=x+i y \in$ $\mathbb{R}^{2}:|y|>\pi / 2$ or $\left.x<0\right\}$, a John disk. Here $w_{j}=R_{D}\left(z_{j}\right)$.

$\operatorname{arcs} \gamma^{*}\left[\zeta^{1}, \zeta^{2}\right]$ and $\gamma^{*}[\xi, \infty]$ intersect in at most one point, however, otherwise these geodesics are the same, contrary to assumption.

Next we show that $R_{D}$ is 1-1. Suppose $w=R_{D}\left(z_{1}\right)=R_{D}\left(z_{2}\right)$. Let $z_{1}=$ $\left(\zeta_{1}^{1}, \xi_{1}, \zeta_{1}^{2}\right), z_{2}=\left(\zeta_{2}^{1}, \xi_{2}, \zeta_{2}^{2}\right)$. Then

$$
\{w\}=\gamma^{*}\left[\infty, \xi_{1}\right] \cap \gamma^{*}\left[\zeta_{1}^{1}, \zeta_{1}^{2}\right]=\gamma^{*}\left[\infty, \xi_{2}\right] \cap \gamma^{*}\left[\zeta_{2}^{1}, \zeta_{2}^{2}\right] ;
$$

hence $\gamma^{*}\left[\infty, \xi_{1}\right]=\gamma^{*}\left[\infty, \xi_{2}\right]$, since these two geodesics now intersect in both $\infty$ and $w$. Furthermore, since $\gamma^{*}\left[\zeta_{1}^{1}, \zeta_{1}^{2}\right]$ and $\gamma^{*}\left[\zeta_{2}^{1}, \zeta_{2}^{2}\right]$ intersect in $D^{*}$, so do $\gamma\left[\zeta_{1}^{1}, \zeta_{1}^{2}\right]$ and $\gamma\left[\zeta_{2}^{1}, \zeta_{2}^{2}\right]$ in $D$. Then $\varphi^{-1}\left(\gamma\left[\zeta_{1}^{1}, \zeta_{1}^{2}\right]\right)$ and $\varphi^{-1}\left(\gamma\left[\zeta_{2}^{1}, \zeta_{2}^{2}\right]\right)$ meet in $\mathbb{H}$. By construction both $\varphi^{-1}\left(\gamma\left[\zeta_{1}^{1}, \zeta_{1}^{2}\right]\right)$ and $\varphi^{-1}\left(\gamma\left[\zeta_{2}^{1}, \zeta_{2}^{2}\right]\right)$ meet $\varphi^{-1}(\gamma[\infty, \xi])$ at right angles, and so they must coincide. Thus $\tilde{z}_{1}=\tilde{z}_{2}$, and $z_{1}=z_{2}$.

To prove that $R_{D}$ is continuous in $\bar{D}$, first take $z \in D$ and consider a sequence $\left(z_{n}\right) \subset D$ with $z_{n} \rightarrow z$. Let $z=\left(\zeta^{1}, \xi, \zeta^{2}\right)$ and $z_{n}=\left(\zeta_{n}^{1}, \xi_{n}, \zeta_{n}^{2}\right)$. Write $w=$ $R_{D}(z), w_{n}=R_{D}\left(z_{n}\right)$. Then $\varphi^{-1}\left(z_{n}\right) \rightarrow \varphi^{-1}(z)$ and

$$
\varphi^{-1}\left(\zeta_{n}^{1}\right) \rightarrow \varphi^{-1}\left(\zeta^{1}\right), \quad \varphi^{-1}\left(\xi_{n}\right) \rightarrow \varphi^{-1}(\xi), \quad \varphi^{-1}\left(\zeta_{n}^{2}\right) \rightarrow \varphi^{-1}\left(\zeta^{2}\right),
$$

as $n \rightarrow \infty$ by continuity of $\varphi$. Thus $\zeta_{n}^{1} \rightarrow \zeta^{1}, \xi_{n} \rightarrow \xi$ and $\zeta_{n}^{2} \rightarrow \zeta^{2}$. Mapping $D^{*}$ onto $\mathbb{H}$ using $\psi^{-1}$, we see that now

$$
\psi^{-1}\left(\zeta_{n}^{1}\right) \rightarrow \psi^{-1}\left(\zeta^{1}\right), \quad \psi^{-1}\left(\xi_{n}\right) \rightarrow \psi^{-1}(\xi), \quad \psi^{-1}\left(\zeta_{n}^{2}\right) \rightarrow \psi^{-1}\left(\zeta^{2}\right),
$$

because $\psi$ is continuous. Thus $\psi^{-1}\left(w_{n}\right) \rightarrow \psi^{-1}(w)$ and finally $w_{n} \rightarrow w$ by continuity once more. For $z \in \partial D$ the same reasoning shows that $w_{n}=R_{D}\left(z_{n}\right) \rightarrow z$ when $z_{n} \rightarrow z$.

Finally we confirm that $R_{D}: D \rightarrow D^{*}$ is onto. It is enough to prove that if $\xi \in \partial D$, then $R_{D}(\gamma[\infty, \xi])=\gamma^{*}[\infty, \xi]$. Because $R_{D}$ is continuous, $R_{D}(\gamma[\infty, \xi])$ is connected. By construction it is clear that there are $w_{n}=R_{D}\left(z_{n}\right) \in \gamma^{*}[\infty, \xi]$ with $w_{n} \rightarrow \infty, n \rightarrow \infty$. Thus $R_{D}(\gamma[\infty, \xi])$ is the whole of $\gamma^{*}[\infty, \xi]$. 
Theorem 4.3. If $\partial D$ is bounded, then $R_{D, p}$ is a reflection for each $p \in \partial D$.

Proof. Apply a Möbius transformation $f: \overline{\mathbb{R}^{2}} \rightarrow \overline{\mathbb{R}^{2}}$ with $f(p)=\infty, f(D)=U$ and $f\left(D^{*}\right)=U^{*}$. Then since $U$ is unbounded with $\infty \in \partial U$,

$$
R_{D, p}=f^{-1} R_{U} f: D \rightarrow D^{*},
$$

where $R_{U}$ is a reflection as in subsection 4.2, and $R_{D, p}$ is a reflection by Theorem 4.2 .

Remark 4.4. In general it is not true that $R_{D^{*}}=R_{D}^{-1}$. This will be explained in Example 6.5.

4.3. Sewing homeomorphisms, reflections and extensions. Here we point out some relations between the reflection $R_{D}$, the sewing homeomorphism associated with $D$ and an extension of this homeomorphism. These ideas will be applied to two examples in Section 6 .

Let $\mathbb{H}_{-}=\{z: \operatorname{Im} z<0\}$ denote the lower half plane. Suppose that $D$ is a Jordan domain with $\infty \in \partial D$. The assumption that $\infty \in \partial D$ is made mostly for convenience, and is not essential for the following discussion.

Consider conformal maps $\varphi: \mathbb{H} \rightarrow D$ and $\psi: \mathbb{H}_{-} \rightarrow D^{*}$ with $\varphi(\infty)=\psi(\infty)=\infty$. We have homeomorphic extensions $\varphi: \overline{\mathbb{H}} \rightarrow \bar{D}$ and $\psi: \overline{\mathbb{H}}_{-} \rightarrow \overline{D^{*}}$, thus inducing an orientation preserving homeomorphism $h=\psi^{-1} \varphi: \mathbb{R} \rightarrow \mathbb{R}$. This is called a sewing homeomorphism associated with $\varphi$ and $\psi$, or a conformal welding of $D$ and $D^{*}$. It relates the geometry of $D$ to that of $D^{*}$. Because $R_{D}$ fixes each point on $\partial D$, we have $h=\psi^{-1} R_{D} \varphi$. This means that the orientation preserving homeomorphism

$$
E_{h}=\overline{\psi^{-1} R_{D} \varphi}: \overline{\mathbb{H}} \rightarrow \overline{\mathbb{H}}
$$

is an extension of the sewing map $h$. By the construction of $R_{D}$, and because $\varphi$ and $\psi^{-1}$ are conformal, and hence map hyperbolic geodesics to hyperbolic geodesics, we may write up an explicit expression for $E_{h}$ in terms of $h$ as follows. For $z=$ $x+i y \in \mathbb{H}$ take the geodesics $\gamma[x-y, x+y]$ and $\gamma[x, \infty]$ in $\mathbb{H}$. They meet at right angles in $z$. Then $E_{h}(z)$ is the point of intersection between $\gamma[h(x-y), h(x+y)]$ and $\gamma[h(x), \infty]$ (see Figure 4). Because $E_{h}(z)$ lies on a half circle with diameter $(h(x-y), h(x+y))$, and the real part of $E_{h}(z)$ is $h(x)$, the imaginary part of $E_{h}(z)$ is just the geometric mean of the two numbers $h(x)-h(x-y)$ and $h(x+y)-h(x)$ :
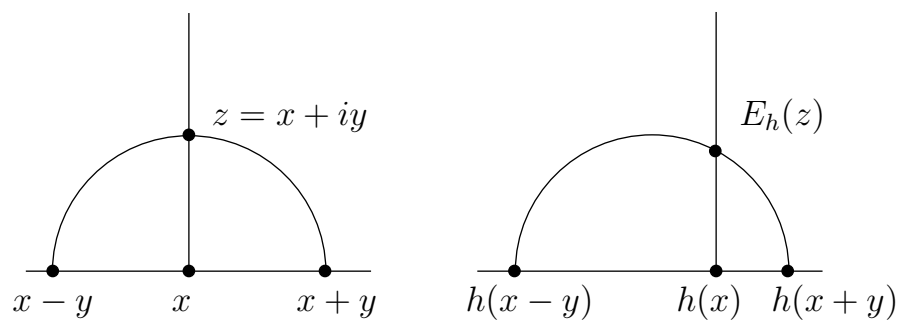

FiguRE 4

$$
E_{h}(z)=h(x)+i \sqrt{(h(x+y)-h(x))(h(x)-h(x-y))} .
$$


Of course $E_{h}$, if defined by (4.6), provides an extension of any homeomorphism $h: \mathbb{R} \rightarrow \mathbb{R}$, not just sewing homeomorphisms.

The extension $E_{h}$ introduced above is similar to the extension used by Tukia and Väisälä in 25. They considered the extension $F_{h}(z)=h(x)+i \tau_{h}(z)$, where $\tau_{h}(z)=\max \{h(x)-h(x-y), h(x+y)-h(x)\}$. The extension $E_{h}$ has a lot of the same properties.

\section{JOHN DISKS AND REFLECTIONS}

We now take a closer look at some specific reflections. Throughout this section $D$ is a Jordan domain in $\overline{\mathbb{R}^{2}}$ with $\infty \in \partial D$, and $R_{D}$ is the reflection as defined in Section 4.2 We use geodesic coordinates $z=\left(\zeta^{1}, \xi, \zeta^{2}\right)$ as in that section too. The goal is to characterize John disks in terms of reflections, and to give a new proof for a result about quasidisks and reflections. First we look at the reflection $R_{D}$ for John disks. Because we are working with Jordan domains, we will write $\partial D$ for the common boundary of $D$ and $D^{*}$.

Lemma 5.1. Suppose that $D$ is a c-John disk with $\infty \in \partial D$. Then there is a constant $L=L(c)$ such that for every $z=\left(\zeta^{1}, \xi, \zeta^{2}\right)$ in $D$,

(a) $\left|R_{D}(z)-\xi\right| \leq L|z-\xi|$;

(b) $\operatorname{dist}\left(R_{D}(z), \partial D\right) \leq c L \operatorname{dist}(z, \partial D)$.

Proof. We prove (a) first. Take any $z \in D$ with $z=\left(\zeta^{1}, \xi, \zeta^{2}\right)$. By Theorem 2.3 we have

$$
\frac{\left|R_{D}(z)-\xi\right|}{|z-\xi|} \leq c_{1} \frac{\left|R_{D}(z)-\xi\right|}{\ell(\gamma[z, \xi])}
$$

Once more a limiting procedure was used, since $\xi \in \partial D$.

Because $D$ is a Jordan John disk, $D^{*}$ is linearly connected [21, Theorem 4.5], so by using Theorem 3.7 we see that there is a constant $c_{2}$ such that

$$
\frac{\left|R_{D}(z)-\xi\right|}{\ell(\gamma[z, \xi])} \leq c_{2} \frac{\max _{j=1,2}\left|\zeta^{j}-\xi\right|}{\lambda_{D}(z, \xi)} .
$$

Of course $\left|\zeta^{j}-\xi\right| \leq \lambda_{D}\left(\zeta^{j}, \xi\right)$. Using Theorem [3.4, we get a constant $c_{3}$ with

$$
\frac{\max _{j=1,2}\left|\zeta^{j}-\xi\right|}{\lambda_{D}(z, \xi)} \leq c_{3} \frac{\max _{j=1,2} \lambda_{D}\left(\zeta^{j}, \xi\right)}{\min _{j=1,2} \lambda_{D}\left(\zeta^{j}, \xi\right)} .
$$

The three inequalities above combine to give

$$
\frac{\left|R_{D}(z)-\xi\right|}{|z-\xi|} \leq c_{4} \frac{\max _{j=1,2} \lambda_{D}\left(\zeta^{j}, \xi\right)}{\min _{j=1,2} \lambda_{D}\left(\zeta^{j}, \xi\right)}
$$

where $c_{4}=c_{1} c_{2} c_{3}$.

Let $\varphi: \mathbb{H} \rightarrow D$ be a conformal map with $\varphi(\infty)=\infty$. Then by Theorem 2.4 the homeomorphism $\varphi: \overline{\mathbb{H}} \rightarrow\left(\bar{D}, \lambda_{D}\right)$ is $\eta$-quasisymmetric, with $\eta$ depending only on $c$. Let $\tilde{\zeta}^{j}, \tilde{\xi} \in \mathbb{R}$ be such that $\zeta^{j}=\varphi\left(\tilde{\zeta}^{j}\right), \xi=\varphi(\tilde{\xi})$. Since by construction $\left|\tilde{\zeta}^{1}-\tilde{\xi}\right|=\left|\tilde{\zeta}^{2}-\tilde{\xi}\right|$, we have

$$
\frac{1}{\eta(1)} \leq \frac{\lambda_{D}\left(\zeta^{1}, \xi\right)}{\lambda_{D}\left(\zeta^{2}, \xi\right)} \leq \eta(1)
$$




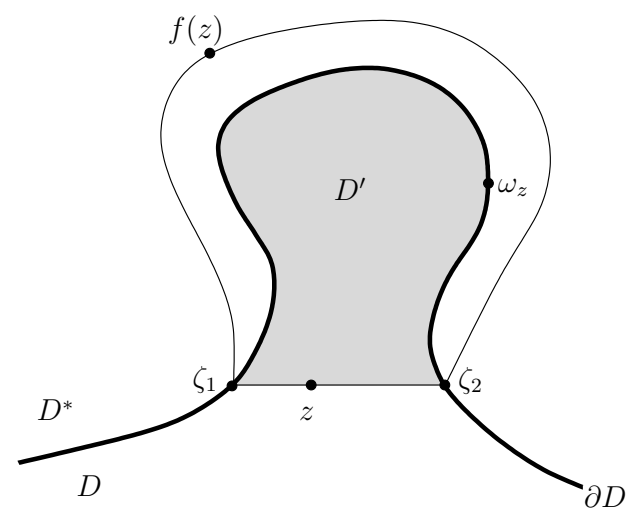

FigURE 5.

Combining this with (5.2) we have finally

$$
\left|R_{D}(z)-\xi\right| \leq L|z-\xi|
$$

where $L=\eta(1) c_{4}$.

To prove (b), note that since $D$ is a $c$-John disk we have by definition (by using $\infty$ as a center)

$$
|z-\xi| \leq \ell(\gamma[z, \xi]) \leq c \operatorname{dist}(z, \partial D) .
$$

Then by (a), $\operatorname{dist}\left(R_{D}(z), \partial D\right) \leq\left|R_{D}(z)-\xi\right| \leq L|z-\xi| \leq c L \operatorname{dist}(z, \partial D)$.

Next we prove a converse of Lemma 5.1

Lemma 5.4. Suppose that $\infty \in \partial D$ and that $\partial D$ admits a reflection $f$ that satisfies the following:

There is a constant $L$ such that for every $z \in D$ there is an $\omega_{z} \in \partial D$ with

$$
\left|f(z)-\omega_{z}\right| \leq L\left|z-\omega_{z}\right| \leq L^{2} \operatorname{dist}(z, \partial D) .
$$

Then $D$ is a c-John disk, with $c$ depending only on $L$.

Proof. We will prove the following. For every straight, or rectilinear, cross cut $\beta=\left(\zeta_{1}, \zeta_{2}\right)$ of $D$, with $\zeta_{1}, \zeta_{2} \in \partial D \backslash\{\infty\}$, one has

$$
\operatorname{diam} D^{\prime} \leq a \operatorname{diam} \beta=a\left|\zeta_{1}-\zeta_{2}\right|,
$$

where $D^{\prime}$ is the bounded component of $D \backslash \beta$. Then $D$ is a $c$-John disk, $c=c(a)$, by Theorem 4.5(4) in 21] (see Figure [5). Let $\zeta_{1}, \zeta_{2} \in \partial D$ with $\beta=\left(\zeta_{1}, \zeta_{2}\right) \subset D$. Then by the assumptions about $f$ and $\omega_{z}$ we have for every $z \in \beta$,

$$
\begin{aligned}
\left|f(z)-\zeta_{1}\right| & \leq\left|f(z)-\omega_{z}\right|+\left|\omega_{z}-\zeta_{1}\right| \\
& \leq L\left|z-\omega_{z}\right|+\left|z-\omega_{z}\right|+\left|z-\zeta_{1}\right| \\
& <L^{2} \operatorname{dist}(z, \partial D)+L \operatorname{dist}(z, \partial D)+\left|\zeta_{1}-\zeta_{2}\right| \\
& <(L+1) L\left|\zeta_{1}-\zeta_{2}\right|+\left|\zeta_{1}-\zeta_{2}\right| \\
& =((L+1) L+1) \operatorname{diam} \beta .
\end{aligned}
$$

Then

$$
\operatorname{diam} D^{\prime} \leq \operatorname{diam}(f(\beta) \cup \beta)=\operatorname{diam} f(\beta) \leq 2((L+1) L+1) \operatorname{diam} \beta .
$$


If $\left(\zeta_{1}, \zeta_{2}\right) \cap D=\emptyset$ for all pairs $\zeta_{1}, \zeta_{2} \in \partial D$, choose any pair of points $\zeta_{1}, \zeta_{2}$ in $\partial D$. We let $\alpha$ be any cross cut from $\zeta_{1}$ to $\zeta_{2}$ in $D$. Then $\alpha \cup\left[\zeta_{1}, \zeta_{2}\right]$ is a Jordan curve. Let $\Omega$ be the bounded component of $\mathbb{R}^{2} \backslash\left(\alpha \cup\left[\zeta_{1}, \zeta_{2}\right]\right)$, and let $D^{\prime}$ be the bounded component of $D \backslash \alpha$. Then we have that

$$
\operatorname{diam} D^{\prime} \leq \operatorname{diam} \Omega=\operatorname{diam}\left(\alpha \cup\left[\zeta_{1}, \zeta_{2}\right]\right)=\operatorname{diam} \alpha .
$$

Thus one may use Theorem 4.5(3) in [21] to conclude that $D$ must be a John disk in this case too.

Combining Lemmas 5.1 and 5.4 we have the following characterization of Jordan John disks (with $\infty \in \partial D$ ). This is the main result of this paper, and a one sided version of Lemma 1 (a) in [1].

Theorem 5.5. Let $D \subset \overline{\mathbb{R}^{2}}$ be a Jordan domain with $\infty \in \partial D$. Then $D$ is a c-John disk if and only if $\partial D$ admits a reflection $f$ such that

$$
|f(z)-\zeta| \leq M|z-\zeta|
$$

for all $z \in D, \zeta \in \partial D \backslash\{\infty\}$. The constants $c$ and $M$ depend only on each other.

Proof. If $\partial D$ admits such a reflection, then $D$ is a $c=c(M)$-John disk by Lemma 5.4. taking $\omega_{z} \in \partial D$ such that $\operatorname{dist}(z, \partial D)=\left|z-\omega_{z}\right|$.

Next suppose that $D$ is a $c$-John disk. Let $z=\left(\zeta^{1}, \xi, \zeta^{2}\right) \in D$ and let $\zeta \in \partial D$. Assume first that $|z-\zeta|<|z-\xi|$. It follows from the triangle inequality that $|\zeta-\xi|<2|z-\xi|$. By (5.3),

$$
|z-\xi| \leq c \operatorname{dist}(z, \partial D) \leq c|z-\zeta| .
$$

Using the above with Lemma 5.1(a) one has

$$
\begin{aligned}
\left|R_{D}(z)-\zeta\right| & \leq\left|R_{D}(z)-\xi\right|+|\zeta-\xi| \\
& <L|z-\xi|+2|z-\xi| \\
& \leq c L|z-\zeta|+2 c|z-\zeta| \\
& =c(L+2)|z-\zeta| .
\end{aligned}
$$

If $|z-\xi| \leq|z-\zeta|$, we have by the triangle inequality $|\xi-\zeta| \leq 2|z-\zeta|$. Then by Lemma 5.1(a):

$$
\begin{aligned}
\left|R_{D}(z)-\zeta\right| & \leq\left|R_{D}(z)-\xi\right|+|\zeta-\xi| \\
& \leq L|z-\xi|+|\zeta-\xi| \\
& \leq 2 L|z-\zeta|+2|z-\zeta| \\
& =2(L+1)|z-\zeta| .
\end{aligned}
$$

Then we can take $f=R_{D}$ and $M=2 c(L+1)$.

We can also use the reflection $R_{D}$ and the results in Section 3 to characterize quasidisks in terms of reflections satisfying a biLipschitz condition.

Lemma 5.6. Suppose that $D \subset \overline{\mathbb{R}^{2}}$ is a $K$-quasidisk with $\infty \in \partial D$. Then for every $z=\left(\zeta^{1}, \xi, \zeta^{2}\right)$ in $D$,

(a) $\frac{1}{L}|z-\xi| \leq\left|R_{D}(z)-\xi\right| \leq L|z-\xi|$;

(b) $\frac{1}{L} \operatorname{dist}(z, \partial D) \leq \operatorname{dist}\left(R_{D}(z), \partial D\right) \leq L \operatorname{dist}(z, \partial D)$.

The constant $L$ depends only on $K$. 
Proof. Since $D$ is a $K$-quasidisk, so is $D^{*}$. Then using Theorem 3.1 for $D$ and $D^{*}$, Remark 3.3 and the construction of $R_{D}$, we have that

$$
\frac{1}{c^{2}} \frac{\min _{j=1,2}\left|\zeta^{j}-\xi\right|}{\max _{j=1,2}\left|\zeta^{j}-\xi\right|} \leq \frac{\left|R_{D}(z)-\xi\right|}{|z-\xi|} \leq c^{2} \frac{\max _{j=1,2}\left|\zeta^{j}-\xi\right|}{\min _{j=1,2}\left|\zeta^{j}-\xi\right|} .
$$

Here $c=c(K)$ is the constant from Theorem 3.1. Now take a conformal map $\varphi: \mathbb{H} \rightarrow D$ fixing $\infty$. Let $\tilde{\zeta}^{j}$ and $\tilde{\xi}$ be preimages in $\mathbb{R}$ of $\zeta^{j}$ and $\xi$ under $\varphi$. By construction (see Section 2.1) we have $\left|\tilde{\zeta}^{1}-\tilde{\xi}\right|=\left|\tilde{\zeta}^{2}-\tilde{\xi}\right|$. Using reflection in $\mathbb{T}$ and Theorem V.3.2 in [19] it is not very difficult to see that $\varphi$ has a $K^{2}$-quasiconformal extension $\varphi: \overline{\mathbb{R}^{2}} \rightarrow \overline{\mathbb{R}^{2}}$. By e.g. Theorem 2.6 in $[9$,

$$
e^{-8 K^{2}} \leq \frac{\left|\zeta^{1}-\xi\right|}{\left|\zeta^{2}-\xi\right|} \leq e^{8 K^{2}}
$$

Now combine (5.7) and (5.8) to get (a) with $L=c^{2} e^{8 K^{2}}$.

Since $D$ is a $K$-quasidisk, both $D$ and $D^{*}$ are $a$-John disks, with $a=a(K)$ [21, Theorem 9.3]. Then it follows as in (5.3) that

$$
|z-\xi| \leq a \operatorname{dist}(z, \partial D) .
$$

Likewise, since $R_{D}(z)$ lies in the geodesic line from $\infty$ to $\xi$, we have

$$
\left|R_{D}(z)-\xi\right| \leq a \operatorname{dist}\left(R_{D}(z), \partial D\right) .
$$

Using these last two inequalities with (a) we have

$$
\frac{1}{L} \operatorname{dist}(z, \partial D) \leq \frac{1}{L}|z-\xi| \leq\left|R_{D}(z)-\xi\right| \leq a \operatorname{dist}\left(R_{D}(z), \partial D\right)
$$

and

$$
\operatorname{dist}\left(R_{D}(z), \partial D\right) \leq\left|R_{D}(z)-\xi\right| \leq L|z-\xi| \leq a L \operatorname{dist}(z, \partial D) .
$$

Thus (b) holds with $M=a L$.

Because $R_{D^{*}}$ is not in general the same as $R_{D}^{-1}$ (see Remark 4.4 and Example 6.5), we could not simply use Lemma 5.1 directly to prove Lemma 5.6. although we do get the upper bound.

Lemma 5.9. Suppose that $\partial D$ admits a reflection $f$ with the following properties: There exists a constant $L$ such that for every $z \in D$ there is an $\omega_{z} \in \partial D$ such that

$$
\frac{1}{L}\left|z-\omega_{z}\right| \leq\left|f(z)-\omega_{z}\right| \leq L\left|z-\omega_{z}\right|
$$

and

$$
\left|z-\omega_{z}\right| \leq L^{2} \operatorname{dist}(z, \partial D), \quad\left|f(z)-\omega_{z}\right| \leq L^{2} \operatorname{dist}(f(z), \partial D) .
$$

Then $D$ is a $K$-quasidisk, with $K=K(L)$.

Proof. The assumptions about $f$ immediately imply, by Lemma [5.4, that $D$ is a John disk. In fact $D^{*}$ is a John disk too, since the inverse of $f$ is a reflection in $\partial D^{*}=\partial D$ satisfying the assumptions of Lemma 5.4 for $D^{*}$. Thus $D$ is a quasidisk by [21, Theorem 9.3]. 
If we combine Lemmas [5.6 and 5.9, we get the following characterization of quasidisks.

Theorem 5.10 ([2]). Let $D \subset \overline{\mathbb{R}^{2}}$ be a Jordan domain with $\infty \in \partial D$. Then $D$ is $a K$-quasidisk if and only if $\partial D$ admits a reflection $f$ such that

$$
\frac{1}{L}|z-\zeta| \leq|f(z)-\zeta| \leq L|z-\zeta|
$$

for all $z \in D, \zeta \in \partial D$, where $K$ and $L$ depend only on each other.

Proof. If $D$ admits a reflection $f$ with the given properties, then Lemma 5.9 shows that $D$ is a quasidisk.

If $D$ is a $K$-quasidisk, then using Lemma 5.6 (for $R_{D}$ and $R_{D}^{-1}$ ) as in the proof of Theorem [5.5] we see that $D$ admits the desired reflection $f=R_{D}$.

Remark 5.11. When taking into account previously known results about reflections and quasidisks, it is perhaps the sufficiency in Theorem 5.10 that is the most interesting, since it is not assumed that the reflection be quasiconformal or biLipschitz. In fact, in [2] Ahlfors only proves the necessity in Theorem 5.10 .

It should also be remembered that all along we have worked completely independent of the Beurling-Ahlfors and Douady-Earle extensions.

Remark 5.12. If $\left|R_{D}(z)-\zeta\right|=|z-\zeta|$ for every $z \in D, \zeta \in \partial D$, then $D$ is a half plane. This can be proved in the same way as Characterization 1.1 in Hag's paper [16. Note that our assumptions are slightly weaker than those in 16] in that we do not assume $R_{D}$ to be an isometry in $D$. Combining this with Example 4.1, we see that $D$ is a half plane if and only if $\partial D$ admits a reflection $f$ such that $|f(z)-\zeta|=|z-\zeta|$ for $z$ in $D$ and $\zeta$ in $\partial D$.

\section{EXAMPLES AND REMARKS}

Here we complement the material in Section 5 by giving counterexamples and providing some further observations regarding the reflections $R_{D}$.

Ahlfors proved that a Jordan domain with $\infty$ on the boundary is a quasidisk if and only if it admits a Euclidean biLipschitz reflection (see e.g. [2, p. 80, Lemma 3] and [11, Theorem 3.6]). Because John disks are often considered to be "one sided" quasidisks (as indicated in [13, 21] and the results in Section 3 of the present paper), Theorem [5.5 might suggest a characterization of John disks in terms of Lipschitz reflections, i.e. reflections with $\left|f\left(z_{1}\right)-f\left(z_{2}\right)\right| \leq L\left|z_{1}-z_{2}\right|$. There are, however, John disks that do not admit Lipschitz reflections. The author is grateful to Christopher J. Bishop for pointing this out by suggesting an example in the same spirit as Example 6.1 below.

Example 6.1. Start with the half plane $D^{\prime}=\{z: \operatorname{Re} z>-8\}$. We obtain a Jordan John domain $D$ by removing a "thickened" snowflake-type Jordan arc from $D^{\prime}$. To be more precise, let $D$ be the component of $D^{\prime} \backslash\left\{\alpha \cup \alpha^{\prime}\right\}$ containing the positive real line, where the arcs $\alpha$ and $\alpha^{\prime}$ are as constructed in Theorem 2.9 in Kim's paper [18. They both end at 0 , and $\alpha \cup \alpha^{\prime}$ is a Jordan arc (see also Figures 1 and 2 in [23]). By Lemma 2.10 in [18], $D$ is a Jordan John domain. At the same time, $0 \in \partial D$ is not rectifiably accessible from $D^{*}=\overline{\mathbb{R}^{2}} \backslash \bar{D}$. If $D$ were to admit a Lipschitz reflection in $\partial D$, then the straight line segment $(0,1]$ in $D$ would map to some rectifiable $\operatorname{arc} \beta$ in $D^{*}$ ending at 0 . This is, however, not possible, since 0 is not rectifiably accessible from $D^{*}$. In short, $\partial D$ admits no Lipschitz reflection. 
Example 6.1 shows that in a sense Theorem [5.5 is the best result of its kind that we can hope to get. We can, however, use Theorem 5.5 to characterize John disks by "coarse" or "rough" Lipschitz reflections. Denote hyperbolic distance in $D$ by $h_{D}$.

Theorem 6.2. Let $D \subset \overline{\mathbb{R}^{2}}$ be a Jordan domain with $\infty \in \partial D$. Then $D$ is a c-John domain if and only if $\partial D$ admits a reflection $f$ such that

$$
\left|f\left(z_{1}\right)-f\left(z_{2}\right)\right| \leq L\left|z_{1}-z_{2}\right|
$$

whenever $z_{1}$ and $z_{2}$ are in $D$ with $h_{D}\left(z_{1}, z_{2}\right) \geq 1$. The constants $L$ and $c$ depend only on each other.

Proof. Let $f$ be a reflection in $\partial D$ with the properties in the theorem. Consider any straight cross cut $\alpha=\left[\zeta_{1}, \zeta_{2}\right]$ of $D$ and take $z \in\left(\zeta_{1}, \zeta_{2}\right)$. By choosing $w_{n} \in\left(\zeta_{1}, z\right)$ with $h_{D}\left(w_{n}, z\right) \geq 1$ (this is possible by choosing each $w_{n}$ close enough to $\zeta_{1}$ ) and such that $w_{n} \rightarrow \zeta_{1}$, we have $\left|f\left(w_{n}\right)-f(z)\right| \leq L\left|w_{n}-z\right|$. Since $f$ is a homeomorphism fixing each boundary point, we have in fact $\left|\zeta_{1}-f(z)\right|=\left|f\left(\zeta_{1}\right)-f(z)\right| \leq L\left|\zeta_{1}-z\right|$. Thus diam $f(\alpha) \leq 2 L\left|\zeta_{1}-\zeta_{2}\right|$, and we see as in the proof of Lemma 5.4 that $D$ is a $c=c(L)$ John domain.

For the converse, assume that $D$ is a c-John domain. Let $\varphi: \mathbb{H} \rightarrow D$ be a conformal map with $\varphi(\infty)=\infty$. Take $z_{1}, z_{2} \in D$ with $h_{D}\left(z_{1}, z_{2}\right) \geq 1, z_{j}=\varphi\left(\tilde{z}_{j}\right)$. Then $h_{\mathbb{H}}\left(\tilde{z}_{1}, \tilde{z}_{2}\right)=h_{D}\left(z_{1}, z_{2}\right)$, and

$$
1 \leq h_{\mathbb{H}}\left(\tilde{z}_{1}, \tilde{z}_{2}\right) \leq \int_{\left[\tilde{z}_{1}, \tilde{z}_{2}\right]} \frac{|d z|}{\operatorname{Im} z} \leq \frac{\left|\tilde{z}_{1}-\tilde{z}_{2}\right|}{\min _{j=1,2} \operatorname{dist}\left(\tilde{z}_{j}, \partial \mathbb{H}\right)} .
$$

Thus $\left|\tilde{z}_{1}-\tilde{z}_{2}\right| \geq \operatorname{dist}\left(\tilde{z}_{1}, \partial \mathbb{H}\right)=\left|\tilde{z}_{1}-\xi\right|$, say. By Theorem 2.4, $\varphi$ is $\eta$-quasisymmetric with respect to internal distances, so we have that $\operatorname{dist}\left(z_{1}, \partial D\right) \leq \lambda_{D}\left(z_{1}, \varphi(\xi)\right) \leq$ $\eta(1) \lambda_{D}\left(z_{1}, z_{2}\right)$. It is not difficult to see that then $\operatorname{dist}\left(z_{1}, \partial D\right) \leq b\left|z_{1}-z_{2}\right|, b=\eta(1)$. Let $f=R_{D}$ and choose $\zeta \in \partial D$ with $\left|z_{1}-\zeta\right|=\operatorname{dist}\left(z_{1}, \partial D\right)$. Then by (the proof of) Theorem 5.5 there is a constant $M=M(c)$ such that

$$
\begin{aligned}
\left|f\left(z_{1}\right)-f\left(z_{2}\right)\right| & \leq\left|f\left(z_{1}\right)-\zeta\right|+\left|f\left(z_{2}\right)-\zeta\right| \\
& \leq M\left|z_{1}-\zeta\right|+M\left|z_{2}-\zeta\right| \\
& \leq M\left|z_{1}-\zeta\right|+M\left(\left|z_{1}-z_{2}\right|+\left|z_{1}-\zeta\right|\right) \\
& \leq 3 M b\left|z_{1}-z_{2}\right| .
\end{aligned}
$$

We may still ask whether $R_{D}$ is always quasiconformal when $D$ is a quasidisk. The answer is no, as shown by the following simple example.

Example 6.3. Let $D=\left\{r e^{i \vartheta}: 0<\vartheta<3 \pi / 2\right\}$ be a sector of angle $3 \pi / 2$ with complementary domain $D^{*}=\left\{r e^{i \vartheta}: 3 \pi / 2<\vartheta<2 \pi\right\}$. Then $D$ is a $K$-quasidisk, with $K=\sqrt{3}$ (see Example 4.1 in [11]). The maps given by $\varphi(z)=z^{3 / 2}$ and $\psi(z)=\overline{\bar{z}^{1 / 2}}$ map $\mathbb{H}$ and $\mathbb{H}_{-}=\{z: y<0\}$ conformally onto $D$ and $D^{*}$, respectively. We see that $h: \mathbb{R} \rightarrow \mathbb{R}, h(x)=x^{3}$, is a sewing homeomorphism associated with $\varphi$ and $\psi$. A calculation using (4.6) shows that

$$
E_{h}(z)=x^{3}+i y \sqrt{9 x^{4}-3 x^{2} y^{2}+y^{4}}, z=x+i y,
$$

where $E_{h}$ is as defined in (4.5). The homeomorphism $h$ is quasisymmetric, but $E=E_{h}$ is not quasiconformal. This can be seen by observing that

$$
\left|\mu_{E}\right|=\left|\frac{E_{\bar{z}}}{E_{z}}\right| \rightarrow 1 \text {, as } x \rightarrow 0 \text { along every horizontal line. }
$$


See 2], Chapter 1, for the background material. Thus $R_{D}=\psi \bar{E}_{h} \varphi^{-1}$ is not quasiconformal either.

The final example explains why we do not have $R_{D^{*}}=R_{D}^{-1}$ in general, thus confirming the claim in Remark 4.4.

Example 6.5. We consider the same situation as in subsection 4.3, Let $h^{*}$ denote the sewing homeomorphism associated with $D^{*}$. Then $h^{*}=\varphi^{-1} \psi=h^{-1}$. By (4.5) we have for $z \in \mathbb{H}$,

$$
E_{h}(z)=\overline{\psi^{-1} R_{D} \varphi(z)}, \quad E_{h^{*}}(z)=\varphi^{-1} R_{D^{*}} \psi(\bar{z}),
$$

which means that

$$
E_{h^{*}} E_{h}(z)=\varphi^{-1} R_{D^{*}} R_{D} \varphi(z) .
$$

Thus $R_{D^{*}}=R_{D}^{-1}$ if and only if $E_{h^{*}} E_{h}(z)=z$.

Now let $D$ be the sector of Example6.3 and let $\varphi, \psi$ and $h$ be as in that example. Then $h(x)=x^{3}$, and $h^{*}(x)=h^{-1}(x)=x^{1 / 3}$. Using (6.4) and (4.6) we have that $E_{h}(1+i)=1+\sqrt{7} i$, while $E_{h^{*}}(1+\sqrt{7} i)=E_{h^{*}} E_{h}(1+i) \approx 1+1.084 i$. Thus $R_{D^{*}} \neq R_{D}^{-1}$ in general.

\section{ACKNOWLEDGMENTS}

This paper is based on Chapter 4 of the author's dr. scient.-thesis [4], written under the supervision of Kari Hag; the author is grateful for all the help and support. The author is also indebted to Frederick W. Gehring who originally suggested studying reflections in John disks. Per Hag carefully read this manuscript and made numerous corrections and suggestions for improvements.

\section{REFERENCES}

1. L. V. Ahlfors, Quasiconformal reflections, Acta Math. 109 (1963), 291-301. MR0154978 $(27: 4921)$

2. L. V. Ahlfors, Lectures on Quasiconformal Mappings, 2nd ed. University Lecture Series 38, American Mathematical Society, 2006. MR2241787

3. A. Beurling and L. V. Ahlfors, The boundary correspondence under quasiconformal mappings, Acta Math. 96 (1956), 125-142. MR0086869 (19:258c)

4. O. J. Broch, Geometry of John disks, Dr. scient.-avhandling, Doctoral Theses at NTNU 2005:21, NTNU, 2005.

5. O. J. Broch, Extension of internally bilipschitz maps in John disks, Ann. Acad. Sci. Fenn. Math. 31 (2006), 13-30. MR2210105 (2006m:30015)

6. A. Douady and C. J. Earle, Conformally natural extension of homeomorphisms of the circle, Acta Math. 157 (1986), 23-48. MR857678 (87j:30041)

7. C. J. Earle and S. Nag, Conformally natural reflections in Jordan curves with applications to Teichmüller spaces, In: Holomorphic Functions and Moduli, II, Math. Sci. Res. Inst. Publ. 11, Springer, 1988, 179-193. MR955840 (89i:30019)

8. F. P. Gardiner and N. Lakic, Quasiconformal Teichmüller Theory, Mathematical Surveys and Monographs 76, American Mathematical Society, 2000. MR1730906 (2001d:32016)

9. F. W. Gehring, Characteristic Properties of Quasidisks, Les presses de l'université de Montréal (1982). MR674294 (84a:30036)

10. F. W. Gehring and K. Hag, Remarks on Uniform and Quasiconformal Extension Domains, Complex Variables 9 (1987), 245-263. MR923218 (89b:30019)

11. F. W. Gehring and K. Hag, Reflections on reflections in quasidisks, Papers on Analysis: A volume dedicated to Olli Martio on the occasion of his 60th birthday. Report. Univ. Jyväskylä. 83 (2001), 81-90. MR1886615 (2003d:30058)

12. F. W. Gehring and K. Hag, Sewing Homeomorphisms and Quasidisks, Comput. Methods Funct. Theory. 3 (2003), 143-150. MR2082011 (2005e:30033) 
13. F. W. Gehring, K. Hag, and O. Martio, Quasihyperbolic geodesics in John domains, Math. Scand. 65 (1989), 75-92. MR.1051825 (91i:30014)

14. F. W. Gehring and W. K. Hayman, An inequality in the theory of conformal mapping, J. Math. Pures Appl. 41 (1962), 353-361. MR0148884 (26:6381)

15. F. W. Gehring and B. Palka, Quasiconformally homogeneous domains, Journal d'Analyse Math. 30 (1976), 172-199. MR0437753 (55:10676)

16. K. Hag, What is a disk? Banach Center Publications. 48 (1999), 43-53. MR:1709973 (2001d:30025)

17. J. Heinonen, Lectures on Analysis on Metric Spaces, Springer 2001. MR.1800917 $(2002 \mathrm{c}: 30028)$

18. K. Kim, Relations between certain domains in the complex plane and polynomial approximation in the domains, Bull. Korean Math. Soc. 39 (2002), 687-704. MR.1939567 (2003i:30011)

19. O. Lehto and K. I. Virtanen, Quasiconformal Mappings in the Plane, 2nd ed. Springer-Verlag 1973. MR0344463 (49:9202)

20. J. Miller, Sector reflections in the plane, Ann. Acad. Sci. Fenn. Math. 30 (2005), 219-225. MR 2173362 (2006e:54029)

21. R. Näkki and J. Väisälä, John disks, Expo. Math. 9 (1991), 3-43. MR1101948 (92i:30021)

22. Ch. Pommerenke, Boundary Behaviour of Conformal Maps, Springer-Verlag, 1992. MR 1217706 (95b:30008)

23. K. Ryu, Properties of John disks, Ph.D. thesis, University of Michigan, 1991.

24. P. Tukia and J. Väisälä, Quasisymmetric embeddings of metric spaces, Ann. Acad. Sci. Fenn. Ser. A I Math. 5 (1980), 97-114. MR595180 (82g:30038)

25. P. Tukia and J. Väisälä, Quasiconformal extension from dimension $n$ to $n+1$, Ann. of Math. 115 (1982), 331-348. MR647809 (84i:30030)

Department of Mathematical Sciences, Norwegian University of Science and TechNOlogy, N-7491 Trondheim, Norway

E-mail address: olejacb@math.ntnu.no 